\title{
Optimal Transient Control of a Heavy Duty Diesel Engine with EGR and VGT
}

\author{
Xavier Llamas* Lars Eriksson* \\ * Vehicular Systems, Dept. of Electrical Engineering, Linköping \\ University, SE-581 83 Linköping, Sweden, \{xavier.llamas.comellas, \\ lars.eriksson\}@liu.se
}

\begin{abstract}
Optimal control of a heavy duty diesel engine with EGR and VGT during transients is investigated. Minimum time and fuel optimal control problems are defined for transients from low to high output torque. A validated diesel engine model is used with minor changes in order to be suitable for the selected solver. The problem is solved for several feasible minimum EGR fractions and smoke-limiter values in order to provide comparisons. The optimization results show that the smoke-limiter has great effect on the transient duration while the required EGR fraction influences the control signals' shape. The fuel optimal control keeps the control actuators more closed than the time optimal, however both time and fuel optimal results become very similar when high EGR fractions and smoke-limiter values are required.
\end{abstract}

Keywords: Simulation, Automotive emissions, Engine systems.

\section{INTRODUCTION}

Reducing diesel engine emissions has become a more and more challenging task every time a new stricter government regulation is approved, such as the new EURO 6 . There are several technologies available to achieve such reductions for heavy duty diesel engines, e.g. exhaust gas recirculation (EGR) systems together with variable geometry turbines (VGT), and exhaust gas aftertreatment systems such as the selective catalytic reduction (SCR). The challenge is thus to control the whole engine together with these complex technologies as optimal as possible.

Much research has been done regarding control of EGR and VGT, e.g. Wahlström (2009) and Nieuwstadt et al. (1998). However few results exist for optimal control of diesel engines. In Sivertsson and Eriksson (2012) optimal control for a diesel-electric powertrain in transient operation is investigated. Solving the optimal control problem (OCP) with a VGT actuator is done in Ekdahl (2005), where a controller for minimizing the difference between the produced torque and the step transient torque is proposed. An example of OCP with EGR and VGT is found in Kolmanovsky et al. (1999), where the objective is to maximize engine speed after a transient.

This study considers a heavy duty diesel engine with EGR and VGT systems, and investigates how it has to be controlled to ensure performance, fuel economy and emission reduction. This is done by solving the OCP for either minimum fuel or time to go from a low load steady state point to a high load steady state point. Engine speed is assumed constant during the transient. The diesel engine model used is the validated mean value engine model (MVEM) presented in Wahlström and Eriksson (2011). This nonlinear model captures the dynamics and the singular characteristics of the diesel engine equipped with EGR and VGT systems. Pseudospectral collocation methods are chosen to solve the OCP, since this approach properly handles the high system complexity and the nonlinearities. Few modifications in the exhaust temperature model are required in order to be solved by the selected software, PROPT (TOMLAB, 2010).

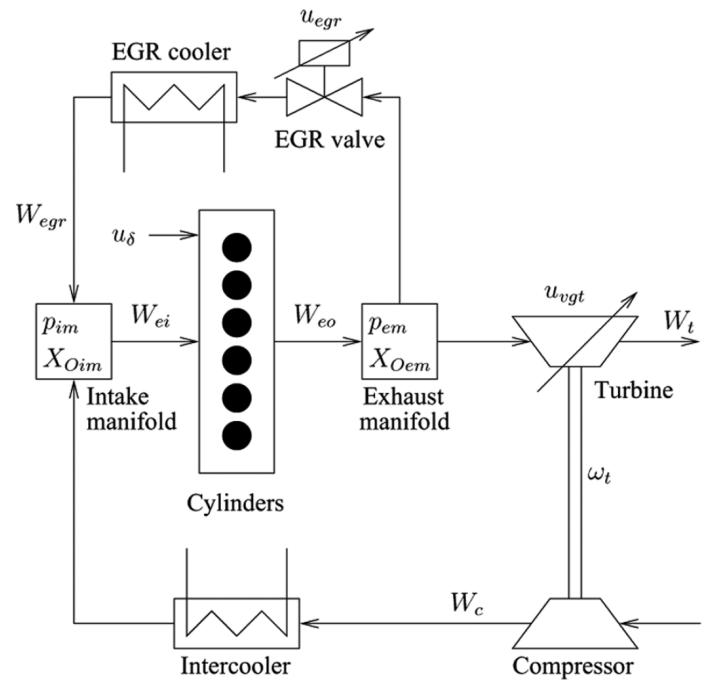

Fig. 1. Structure of the modeled diesel engine. It presents state variables, control inputs and mass flows.

\section{ENGINE MODEL}

The modeled engine is a 6 cylinder 12.7 liter SCANIA diesel engine with VGT and EGR systems. More information about the model, engine components, equations, parameters and nomenclature can be found in Wahlström and Eriksson (2011). The five states of the MVEM are intake manifold pressure, $\mathrm{p}_{\mathrm{im}}$, exhaust manifold pressure, $\mathrm{p}_{\mathrm{em}}$, intake manifold oxygen concentration, $X_{\text {Oim }}$, exhaust manifold oxygen concentration, $X_{\text {Oem }}$, and turbocharger speed, $\omega_{\mathrm{t}}$. The controls are injected fuel mass, $\mathrm{u}_{\delta}, \mathrm{EGR}$ valve position, $\mathrm{u}_{\mathrm{egr}}$, and VGT actuator position, $\mathrm{u}_{\mathrm{vgt}}$. The intake and exhaust manifolds are modeled as control 
volumes with a standard isothermal model based on the ideal gas law and mass conservation. The oxygen concentrations in the manifolds are modeled as dynamic systems by differentiating its physical definition $\left(X_{\mathrm{O}}=\frac{m_{\mathrm{O}}}{m_{\mathrm{to}}}\right)$ and using mass conservation. The turbocharger speed is modeled using Newton's second law. Hence, the differential equations that control the evolution of the MVEM states are:

$$
\begin{aligned}
\frac{\mathrm{d}}{\mathrm{d} t} \mathrm{p}_{\mathrm{im}} & =\frac{R_{\mathrm{a}} T_{\mathrm{im}}}{V_{\mathrm{im}}}\left(W_{\mathrm{c}}+W_{\mathrm{egr}}-W_{\mathrm{ei}}\right) \\
\frac{\mathrm{d}}{\mathrm{d} t} \mathrm{p}_{\mathrm{em}} & =\frac{R_{\mathrm{e}} T_{\mathrm{em}}}{V_{\mathrm{em}}}\left(W_{\mathrm{eo}}-W_{\mathrm{t}}-W_{\mathrm{egr}}\right) \\
\frac{\mathrm{d}}{\mathrm{d} t} X_{\mathrm{Oim}} & =\frac{R_{\mathrm{a}} T_{\mathrm{im}}}{\mathrm{p}_{\mathrm{im}} V_{\mathrm{im}}}\left(\left(X_{\mathrm{Oem}}-X_{\text {Oim }}\right) W_{\mathrm{egr}}+\left(X_{\mathrm{Oc}}-X_{\text {Oim }}\right) W_{\mathrm{c}}\right) \\
\frac{\mathrm{d}}{\mathrm{d} t} X_{\mathrm{Oem}} & =\frac{R_{\mathrm{e}} T_{\mathrm{em}}}{\mathrm{p}_{\mathrm{em}} V_{\mathrm{em}}}\left(\left(X_{\mathrm{Oe}}-X_{\text {Oem }}\right) W_{\mathrm{eo}}\right) \\
\frac{\mathrm{d}}{\mathrm{d} t} \omega_{\mathrm{t}} & =\frac{P_{\mathrm{t}} \eta_{\mathrm{m}}-P_{\mathrm{c}}}{J_{\mathrm{t}} \omega_{\mathrm{t}}}
\end{aligned}
$$

Where $W$ stands for mass flow, $P$ for power, $R$ for gas constant, $V$ for volume, $J$ for inertia, $T$ for temperature and $\eta$ for efficiency. $X_{\mathrm{Oc}}$ and $X_{\mathrm{Oe}}$ refer to oxygen concentration on compressor and cylinder outlets respectively. Figure 1 presents the relations between the engine model components which are described in the following sections.

\subsection{Cylinder}

The cylinder is described using three submodels. First the mass flow model describes the air and fuel mass flows $\left(W_{\mathrm{ei}}\right.$ and $\left.W_{\mathrm{f}}\right)$, volumetric efficiency $\left(\eta_{\mathrm{vol}}\right)$, oxygen-to-fuel ratio $\left(\lambda_{\mathrm{O}}\right)$ as well as the oxygen concentration out of the cylinder $\left(X_{\mathrm{Oe}}\right)$.

$$
\begin{aligned}
W_{\mathrm{ei}} & =\frac{\eta_{\mathrm{vol}} \mathrm{p}_{\mathrm{im}} \mathrm{n}_{\mathrm{e}} V_{\mathrm{d}}}{120 R_{\mathrm{a}} T_{\mathrm{im}}} \\
W_{\mathrm{f}} & =\frac{10^{-6}}{120} \mathrm{u}_{\delta} \mathrm{n}_{\mathrm{e}} n_{\mathrm{cyl}} \\
\eta_{\mathrm{vol}} & =c_{\mathrm{vol} 1 \sqrt{\mathrm{p}_{\mathrm{im}}}+c_{\mathrm{vol} 2} \sqrt{\mathrm{n}_{\mathrm{e}}}+c_{\mathrm{vol} 3}} \\
X_{\mathrm{Oe}} & =\frac{W_{\mathrm{ei}} X_{\mathrm{Oim}}-W_{\mathrm{f}}(O / F)_{\mathrm{s}}}{W_{\mathrm{eo}}} \\
\lambda_{\mathrm{O}} & =\frac{W_{\mathrm{ei}} X_{\mathrm{Oim}}}{W_{\mathrm{f}}(O / F)_{\mathrm{s}}}
\end{aligned}
$$

As it is done in Sivertsson and Eriksson (2012), to avoid problems when $W_{\mathrm{f}}=0$ a new variable is defined

$$
\phi_{\lambda_{\mathrm{O}}}\left(\lambda_{\mathrm{O}, \min }\right)=W_{\mathrm{ei}} X_{\mathrm{Oim}}-\lambda_{\mathrm{O}, \min } W_{\mathrm{f}}(O / F)_{\mathrm{s}}
$$

Then the engine torque $\left(M_{\mathrm{e}}\right)$ is modeled using three torque terms and an ignition efficiency model.

$$
\begin{aligned}
M_{\mathrm{e}} & =M_{\mathrm{ig}}-M_{\mathrm{p}}-M_{\text {fric }} \\
M_{\mathrm{ig}} & =\frac{\mathrm{u}_{\delta} 10^{-6} n_{\mathrm{cyl}} \eta_{\mathrm{ig}} q_{\mathrm{HV}}}{4 \pi} \\
M_{\mathrm{p}} & =\frac{V_{\mathrm{d}}}{4 \pi}\left(\mathrm{p}_{\mathrm{em}}-\mathrm{p}_{\mathrm{im}}\right) \\
M_{\text {fric }} & =\frac{V_{\mathrm{d}} 10^{5}}{4 \pi}\left(c_{\text {fric } 1}\left(\frac{\mathrm{n}_{\mathrm{e}}}{1000}\right)^{2}+c_{\text {fric } 2}\left(\frac{\mathrm{n}_{\mathrm{e}}}{1000}\right)+c_{\text {fric } 3}\right) \\
\eta_{\text {ig }} & =\eta_{\text {igch }}\left(1-\frac{1}{r_{\mathrm{c}}^{\gamma_{\text {cyl }}-1}}\right)
\end{aligned}
$$

Third the engine exhaust manifold temperature is a simplified version of the the model proposed in Wahlström and Eriksson (2011). The assumption is that the residual gas fraction do not affect the temperature when the inlet valve closes, and thus the exhaust temperature can be computed straightforward without fixed point iteration. This is done because the OCP solver cannot handle the original fixedpoint iteration model.

$$
\begin{aligned}
q_{\text {in }}= & \frac{W_{\mathrm{f}} q_{\mathrm{HV}}}{W_{\mathrm{ei}}+W_{\mathrm{f}}} \\
x_{\mathrm{p}}= & 1+\frac{q_{\mathrm{in}} x_{\mathrm{cv}}}{c_{\mathrm{va}} T_{\mathrm{im}} r_{\mathrm{c}}^{\gamma_{\mathrm{a}}-1}} \\
T_{\mathrm{e}}= & \eta_{\mathrm{sc}} \Pi_{\mathrm{e}}^{1-\frac{1}{\gamma_{\mathrm{a}}}} r_{\mathrm{c}}^{1-\gamma_{\mathrm{a}}} x_{\mathrm{p}}^{\frac{1}{\gamma_{\mathrm{a}}}-1} \\
& \left(q_{\mathrm{in}}\left(\frac{1-x_{\mathrm{cv}}}{c_{\mathrm{pa}}}+\frac{x_{\mathrm{cv}}}{c_{\mathrm{va}}}\right)+T_{\mathrm{im}} r_{\mathrm{c}}^{\gamma_{\mathrm{a}}-1}\right) \\
T_{\mathrm{em}}= & T_{\mathrm{amb}}+\left(T_{\mathrm{e}}-T_{\mathrm{amb}}\right) \mathrm{e}^{-\frac{h_{\mathrm{tot}} \pi d_{\mathrm{pipe}} l_{\mathrm{pipe}} n_{\mathrm{pipe}}}{W_{\mathrm{eoo}} c_{\mathrm{pe}}}}
\end{aligned}
$$

\subsection{Compressor}

The compressor consists of a submodel for the compressor mass flow and another submodel for the required power. The mass flow is described by the following equations.

$$
\begin{aligned}
\Psi_{\mathrm{c}} & =\frac{2 c_{\mathrm{pa}} T_{\mathrm{amb}}\left(\Pi_{\mathrm{c}}^{1-\frac{1}{\gamma_{\mathrm{a}}}}-1\right)}{R_{\mathrm{c}}^{2} \omega_{\mathrm{t}}^{2}} \\
c_{\Psi 1}\left(\omega_{\mathrm{t}}\right) & =c_{\omega \Psi 1} \omega_{\mathrm{t}}^{2}+c_{\omega \Psi 2} \omega_{\mathrm{t}}+c_{\omega \Psi 3} \\
c_{\Phi 1}\left(\omega_{\mathrm{t}}\right) & =c_{\omega \Phi 1} \omega_{\mathrm{t}}^{2}+c_{\omega \Phi 2} \omega_{\mathrm{t}}+c_{\omega \Phi 3} \\
\Phi_{\mathrm{c}} & =\sqrt{\max \left(0, \frac{1-c_{\Psi 1} \Psi_{\mathrm{c}}^{2}}{c_{\Phi 1}}\right)} \\
W_{\mathrm{c}} & =\frac{p_{\mathrm{amb}} \pi R_{\mathrm{c}}^{3} \omega_{\mathrm{t}}}{R_{\mathrm{a}} T_{\mathrm{amb}}} \Phi_{\mathrm{c}}
\end{aligned}
$$

The compressor required power is modeled as follows using a compressor efficiency model.

$$
\begin{aligned}
& \eta_{\mathrm{c}}=\eta_{\mathrm{cmax}}-\left[\begin{array}{c}
W_{\mathrm{c}}-W_{\mathrm{copt}} \\
\pi_{\mathrm{c}}-\pi_{\mathrm{copt}}
\end{array}\right]^{T} \mathbf{Q}_{\mathrm{c}}\left[\begin{array}{c}
W_{\mathrm{c}}-W_{\mathrm{copt}} \\
\pi_{\mathrm{c}}-\pi_{\mathrm{copt}}
\end{array}\right] \\
& \pi_{\mathrm{c}}=\left(\Pi_{\mathrm{c}}-1\right)^{c_{\pi}} \\
& P_{\mathrm{c}}=\frac{W_{\mathrm{c}} c_{\mathrm{pa}} T_{\mathrm{amb}}\left(\Pi_{\mathrm{c}}^{1-\frac{1}{\gamma_{\mathrm{a}}}}-1\right)}{\eta_{\mathrm{c}}}
\end{aligned}
$$

\subsection{Turbine}

The turbine consists of a submodel for the turbine mass flow, which depends on the VGT control signal, and another submodel for the produced power. The turbine mass flow is determined using the following equations.

$$
\begin{aligned}
f_{\Pi \mathrm{t}}\left(\Pi_{\mathrm{t}}\right) & =\sqrt{1-\Pi_{\mathrm{t}}^{K_{\mathrm{t}}}} \\
f_{\mathrm{vgt}}\left(\mathrm{u}_{\mathrm{vgt}}\right) & =c_{\mathrm{f} 2}+c_{\mathrm{f} 2} \sqrt{\max \left(0,1-\left(\frac{\mathrm{u}_{\mathrm{vgt}}-c_{\mathrm{vgt} 2}}{c_{\mathrm{vgt} 1}}\right)^{2}\right)} \\
W_{\mathrm{t}} & =\frac{A_{\mathrm{vgtmax}} \mathrm{p}_{\mathrm{em}} f_{\Pi \mathrm{t}}\left(\Pi_{\mathrm{t}}\right) f_{\mathrm{vgt}}\left(\mathrm{u}_{\mathrm{vgt}}\right)}{\sqrt{T_{\mathrm{em}} R_{\mathrm{e}}}}
\end{aligned}
$$

The turbine produced power as well as the turbine efficiency model are defined by the next equations.

$$
\begin{aligned}
c_{\mathrm{m}} & =c_{\mathrm{m} 1}\left[\max \left(0, \omega_{\mathrm{t}}-c_{\mathrm{m} 2}\right)\right]^{c_{\mathrm{m} 3}} \\
\mathrm{BSR} & =\frac{R_{\mathrm{t}} \omega_{\mathrm{t}}}{\sqrt{2 c_{\mathrm{pe}} T_{\mathrm{em}}\left(1-\Pi_{\mathrm{t}}^{1-\frac{1}{\gamma_{\mathrm{e}}}}\right)}} \\
\eta_{\mathrm{tm}} & =\eta_{\mathrm{tm}, \max }-c_{\mathrm{m}}\left(\mathrm{BSR}-\mathrm{BSR}_{\mathrm{opt}}\right)^{2} \\
P_{\mathrm{t}} & =W_{\mathrm{t}} \eta_{\mathrm{tm}} c_{\mathrm{pe}} T_{\mathrm{em}}\left(1-\Pi_{\mathrm{t}}^{1-\frac{1}{\gamma_{\mathrm{e}}}}\right)
\end{aligned}
$$

\subsection{EGR valve}

The EGR valve is considered to be a compressible flow restriction with variable area. The used model equations 
are as follows.

$$
\begin{aligned}
& \Psi_{\mathrm{egr}}=1-\left(\frac{1-\Pi_{\mathrm{egr}}}{1-\Pi_{\mathrm{egropt}}}-1\right)^{2} \\
& f_{\text {egr }}=\min \left(c_{\text {egr } 1} \mathrm{u}_{\text {egr }}^{2}+c_{\text {egr } 2} \mathrm{u}_{\text {egr }}+c_{\text {egr } 3}, c_{\text {egr } 3}-\frac{c_{\text {egr } 2}^{2}}{4 c_{\text {egr } 1}}\right) \\
& W_{\text {egr }}=\frac{A_{\text {egrmax }} f_{\text {egr }} \mathrm{p}_{\text {em }} \Psi_{\text {egr }}}{\sqrt{T_{\text {em }} R_{\mathrm{e}}}} \\
& x_{\text {egr }}=\frac{W_{\text {egr }}}{W_{\mathrm{c}}+W_{\text {egr }}}
\end{aligned}
$$

The EGR pressure ratio is limited with the next relation.

$$
\Pi_{\text {egr }}= \begin{cases}\Pi_{\text {egropt }} & \text { if } \frac{p_{\text {im }}}{p_{\text {em }}}<\Pi_{\text {egropt }} \\ \frac{p_{\text {im }}}{p_{\text {em }}} & \text { if } \Pi_{\text {egropt }} \leq \frac{p_{\text {im }}}{p_{\text {em }}} \leq 1 \\ 1 & \text { if } 1<\frac{p_{\text {im }}}{p_{\text {em }}}\end{cases}
$$

\section{PROBLEM FORMULATION}

The studied transients start from steady state with medium engine speed and low torque, and finish at a higher steady state torque value. The objective is to find the controls that minimize fuel and time using the non-linear engine model described in Section 2. These two OCPs are formulated as:

$$
\begin{array}{ll}
\min & \int_{0}^{T} W_{\mathrm{f}} \mathrm{dt} \quad \text { or } \min T \\
\text { s.t. } & \dot{x}=f(x, u)
\end{array}
$$

where $x$ is the system state and $\dot{x}$ is determined by (1) - (5). The problem constraints are defined by the initial and final conditions of the studied transient, the values are presented in Table 1. Moreover in order to evaluate the effects of the EGR fraction on the cylinder as well as of the minimum oxygen to fuel ratio $\left(\lambda_{\mathrm{O}, \min }\right)$ in the smokelimiter (11), additional constraints are applied to the OCP. The problem constraints are:

$$
\begin{array}{lr}
x(0)=x_{o}, & \dot{x}(0)=0 \\
M_{\mathrm{e}}(0)=M_{\mathrm{e}_{o}}, & M_{\mathrm{e}}(T)=M_{\mathrm{e}_{T}} \\
\dot{M}_{\mathrm{e}} \geq 0, & \dot{x}(T)=0 \\
\phi_{\lambda_{\mathrm{O}}}\left(\lambda_{\mathrm{O}, \min }\right) \geq 0, & x_{\mathrm{egr}} \geq x_{\mathrm{egr}, \min }
\end{array}
$$

Hence solving the OCP defined in (41)-(42) will give either the time or the fuel optimal control signals to reach the requested torque with different EGR and lambda requirements. Note that the engine speed is not a state of the engine model, instead it is considered as a given input in the original model. For simplicity and considering a large inertia, the engine speed is considered to be constant and equal to $1125 \mathrm{rpm}$.

Table 1. Initial and final condition values.

\begin{tabular}{llll}
\hline \hline Variable & Value & Variable & Value \\
\hline$p_{\mathrm{im}_{o}}$ & $120.6 \mathrm{kPa}$ & $p_{\mathrm{em}_{o}}$ & $137.2 \mathrm{kPa}$ \\
$X_{\mathrm{Oim}_{o}}$ & $0.2118-$ & $X_{\mathrm{Oem}_{o}}$ & $0.1596-$ \\
$\omega_{\mathrm{t}_{o}}$ & $4096 \mathrm{rad} / \mathrm{s}$ & $M_{\mathrm{e}_{o}}$ & $250 \mathrm{Nm}$ \\
$M_{\mathrm{e}_{T}}$ & $2000 \mathrm{Nm}$ & & \\
\hline
\end{tabular}

\section{PROBLEM SOLVING}

Pseudospectral collocation methods are direct methods for solving OCPs, which have the feature to represent the state trajectories on the integration interval as high order polynomials. This technique is capable to transform efficiently the OCP into a nonlinear program while maintaining high integration accuracy. Several papers exist that describe the method, e.g. Garg et al. (2010). The problem is complex and difficult to solve, where the numerical solver fails to find a solution given an arbitrary initial guess. To remedy this a method for construction initial guesses by solving a sequence of reduced problems is introduced.

First, feasible initialization data is obtained by solving the OCP with a simplified version of the model in section 2. This simplification consists of reducing the volumetric, the compressor and the turbine efficiencies to constants. Thus (8), (26), (27), (32), (33) and (34) are substituted by $\eta_{\mathrm{vol}}=0.9, \eta_{\mathrm{c}}=0.6$ and $\eta_{\mathrm{tm}}=0.5$. Then the OCP is solved and when it converges, the solution is used as initial guess for the OCP with the volumetric efficency model included. Again, the obtained result is used as initial guess to solve the OCP with the compressor and the volumetric efficiency models included. Finally following the same idea, the previous solution is used as initial guess for the OCP with the complete model.

The OCP complexity is reduced by first solving the OCP with the constraints, (42), only fulfilled in the collocation points. The results are often oscillatory and do not fulfill the constraints between the collocation points, e.g. the EGR fraction tends to oscillate around $x_{\mathrm{egr} \text { min }}$. However these intermediate results are useful as initialization for the OCP with the constraints extended to points in between the collocation points. This ensures that the problem constraints are satisfied. The number of collocation points used is also increased progressively to refine the solution.
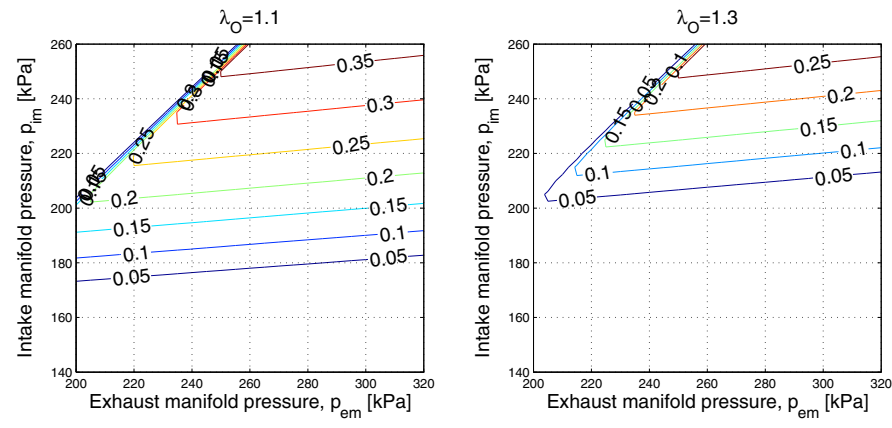

Fig. 2. Maximum EGR fractions at steady state for two lambda values as funcition of manifold pressures. At $1125 \mathrm{rpm}$ and $2000 \mathrm{Nm}$ output torque.

Lower limits for lambda and EGR fraction were introduced in Section 3. An investigation is carried out to find feasible lower limits for the transient final requested torque. Figure 2 depicts the maximum EGR fractions at steady state conditions in order to produce the desired final torque. The intake manifold pressure has a big effect on the EGR fraction achievable. Note that the top left corner in both graphs in Figure 2 is not feasible since the back pressure must be higher than the intake pressure to provide EGR flow. Thus, minimum lambda and EGR fraction values are selected according to these limitations, the values are: $x_{\text {egr,min }}=\left[\begin{array}{lll}0.15, & 0.20, & 0.25\end{array}\right]$ and $\lambda_{\mathrm{O}, \min }=$ $[1.1,1.2,1.3]$. 
If high EGR and lambda values are required, the convergence of the solution is slow and often the control signals present high frequency oscillations or pronounced peaks. In order to reduce this undesired behavior, the sum of the squared control signals derivatives multiplied by a penalty factor is added to the objective functions, see $(43)-(44)$. The OCP is then iteratively solved starting with large $k_{\mathrm{p}}$ values that are progressively decreased, the iteration stops for the lowest $k_{\mathrm{p}}$ that gives smooth controls. A suitable initial $k_{\mathrm{p}}$ value is the one that properly weights the control signal oscillation term against the original objective function (41). For this study a suitable starting value is found to be $k_{\mathrm{p}}=1 \cdot 10^{-4}$. Using this technique, a smoothed version of the original control signals is obtained. The minimum fuel case has a maximum $0.5 \%$ increase of fuel consumption using the technique, while the minimum time case has a $0.6 \%$ increase in time compared to the case with $k_{\mathrm{p}}=0$.

$$
\begin{gathered}
\min \int_{0}^{T} W_{\mathrm{f}} \mathrm{dt}+k_{\mathrm{p}}\left(\int_{0}^{T} \dot{u}_{\mathrm{egr}}^{2} \mathrm{dt}+\int_{0}^{T} \dot{u}_{\mathrm{vgt}}^{2} \mathrm{dt}\right) \\
\min T+k_{\mathrm{p}}\left(\int_{0}^{T} \dot{u}_{\mathrm{egr}}^{2} \mathrm{dt}+\int_{0}^{T} \dot{u}_{\mathrm{vgt}}^{2} \mathrm{dt}\right)
\end{gathered}
$$

\section{RESULTS}

Results of the OCP (41) - (44), with different lambda and EGR limits are described and discussed in the following sections.

\subsection{Fuel optimal transients}

Fuel optimal controls without EGR fraction requirements are shown in Figure 3. The optimal policy is to keep both actuators closed. Keeping VGT closed increases the back pressure and accelerates the turbocharger to build up intake pressure. This is maintained until the intake manifold pressure is high enough to burn the required amount of fuel for the transient end conditions, while satisfying the minimum lambda requirement. At the very end of the transient one can observe that the VGT is opened slightly to decrease the back pressure and reduce the pumping losses, allowing the engine to reach the final torque request. The control behavior is similar for each $\lambda_{\mathrm{O}, \mathrm{min}}$ value. The major change is that a higher lambda limit implies more intake pressure, thus the length of the transient increases in order to build up the required intake pressure.

When certain EGR fraction is required, the fuel optimal strategy is still to keep the VGT actuator at its lowest value. However the EGR valve is kept to a value that gives the desired $x_{\mathrm{egr}}$. At the end of the transient EGR is opened to satisfy the $x_{\text {egr }}$ constraint. Note that the exhaust pressure cannot go below the intake pressure as in the previous case because a certain EGR flow is required. Results for $x_{\text {egr,min }}=0.20$ are depicted for each $\lambda_{\mathrm{O} \text {,min }}$ in Figure 4 . For the case $x_{\mathrm{egr}, \mathrm{min}}=0.15$, the EGR valve position is slightly more closed than the $x_{\text {egr,min }}=0.20$ case, but the same control strategy is observed.

Some differences arise for $x_{\mathrm{egr}, \min }=0.25$ and $\lambda_{\mathrm{O}, \min }=1.3$, see Figure 5 . The time required to finish this transient with the highest lambda and EGR values increases substantially with respect to the one for $\lambda_{\mathrm{O}, \min }=1.2$, but the control strategy is still very similar.

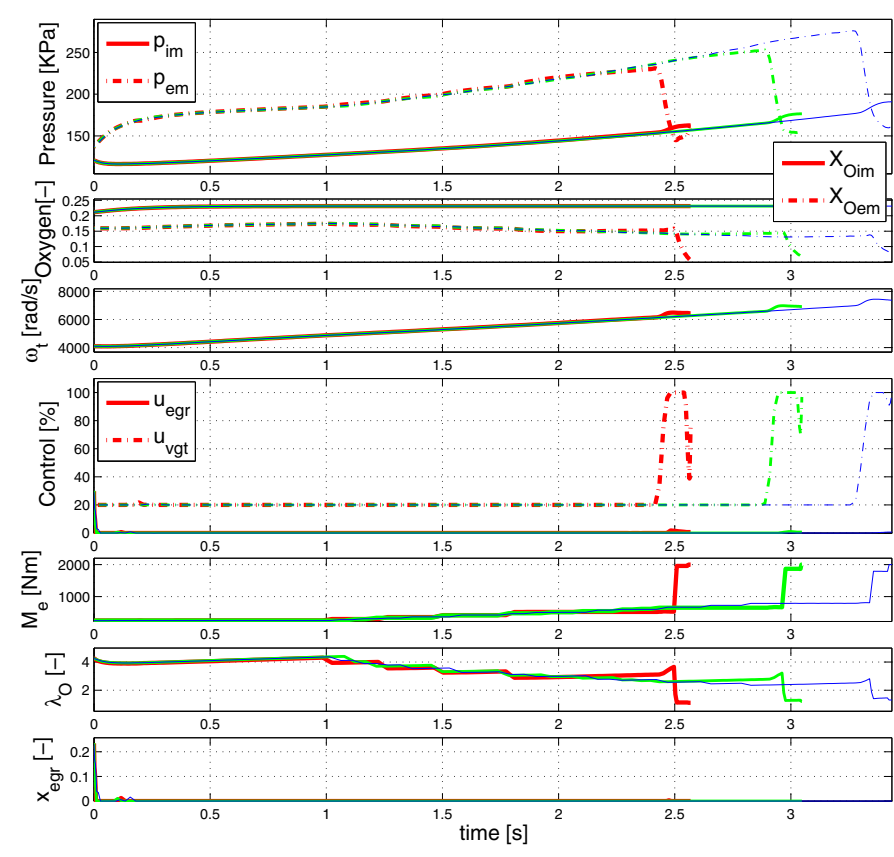

Fig. 3. Fuel optimal results without EGR limit. Red, green and blue are respectively $\lambda_{\mathrm{O}, \min }=[1.1,1.2,1.3]$.

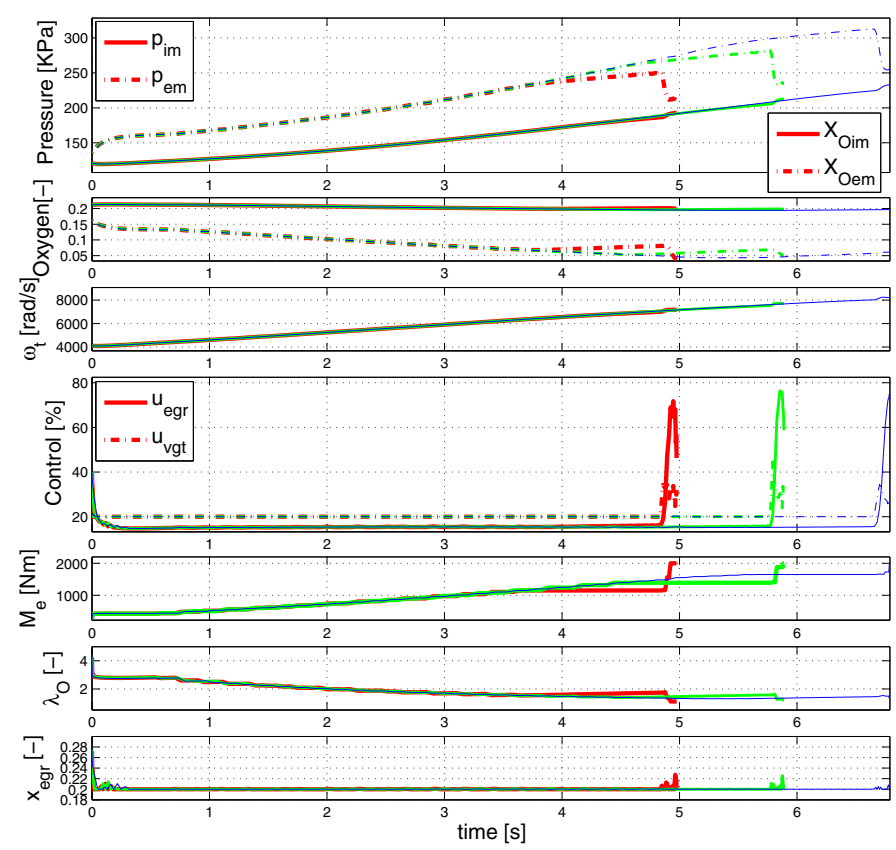

Fig. 4. Fuel optimal results for $x_{\text {egr,min }}=0.20$. Red, green and blue are respectively $\lambda_{\mathrm{O}, \min }=[1.1,1.2,1.3]$.

\subsection{Time optimal transients}

Figure 6 presents the time optimal results without required EGR fraction. The optimal strategy is again to close both VGT and EGR actuators. Moreover, the injected fuel control signal is always at its maximum value, which sets the lambda signal at its lower limit. This is done to accelerate the turbocharger faster, and thus reduce the transient time by speeding up the intake pressure increase. Intuition would indicate that completely closing the VGT like in the fuel optimal case would reduce the transient time. However by fully closing the VGT, the back pressure 


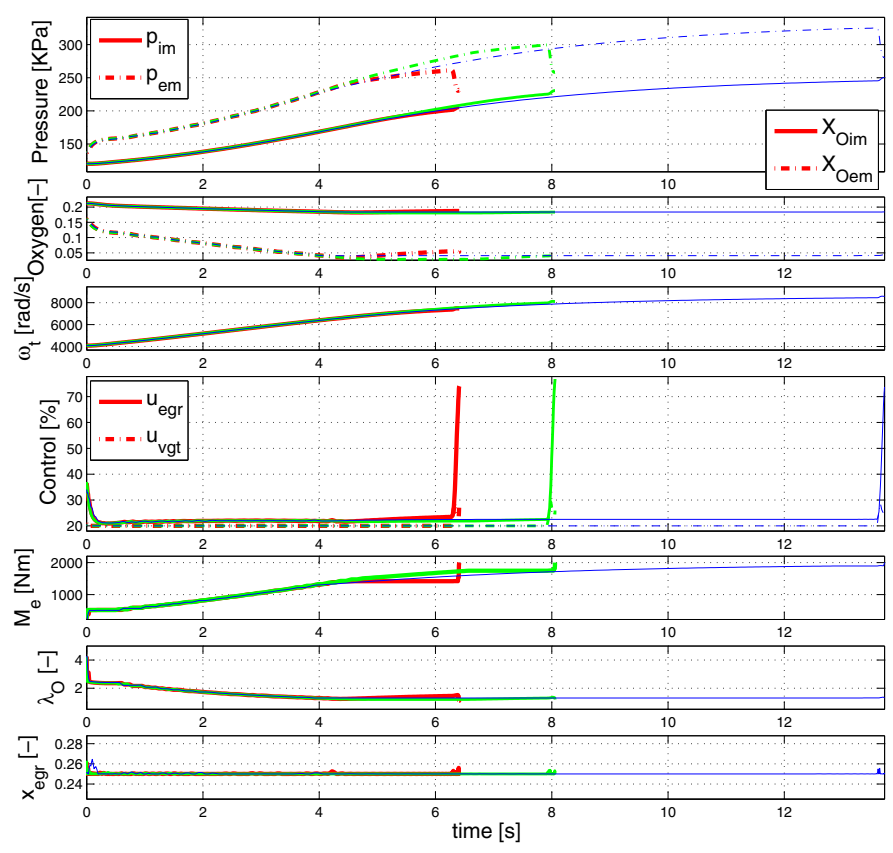

Fig. 5. Fuel optimal results for $x_{\text {egr,min }}=0.25$. Red, green and blue are respectively $\lambda_{\mathrm{O}, \min }=[1.1,1.2,1.3]$.

and temperature are highly increased due to the high fuel injection signal, this reduces a lot the BSR value, (33), which yields to a very low turbine efficiency value. Hence the time optimal strategy is not to fully close the VGT, instead it is better to gradually close the VGT to keep a lower back pressure. Furthermore, the torque signal is smoother than in the fuel optimal case, e.g. there is no final peak. At the end of the transient, the VGT actuator is opened to reduce the back pressure and thus reduce the pumping losses to produce the desired final torque.

When EGR fraction is required, the VGT actuator is still progressively closed while now the EGR valve is instead controlled to produce the desired $x_{\mathrm{egr}}$ level. Similar to the fuel optimal case the EGR is opened at the transient end to ensure the EGR level. Note again that the exhaust pressure does not go below the intake pressure in order to fulfill the EGR fraction requirements. This reasoning applies for the cases $x_{\text {egr,min }}=0.15$ and $x_{\text {egr,min }}=0.20$ with differences in the EGR valve position to fulfill the different EGR fraction requirements. Furthermore if higher EGR fraction is required, the transient time is consequently increased. Results for $x_{\mathrm{egr} \text {,min }}=0.20$ and each $\lambda_{\mathrm{O} \text {,min }}$ value are shown in Figure 7.

Results for the highest EGR fraction are depicted in Figure 8. Where, as observed in the fuel optimal results, the $\lambda_{\mathrm{O}, \min }=1.3$ case requires a much longer transient time to reach the requested torque compared to the $\lambda_{\mathrm{O}, \min }=1.2$ case.

\subsection{Effects of $\lambda_{O, \min }$ and $x_{\text {egr,min. }}$.}

Analyzing the results presented in the sections 5.1 and 5.2 some conclusions can be extracted. First, the major effect of increasing $\lambda_{\mathrm{O}, \min }$ is the increase of the transient time both in the time and fuel optimal problems. On the other hand $x_{\text {egr,min }}$ changes the $\mathrm{u}_{\text {egr }}$ position depending if more

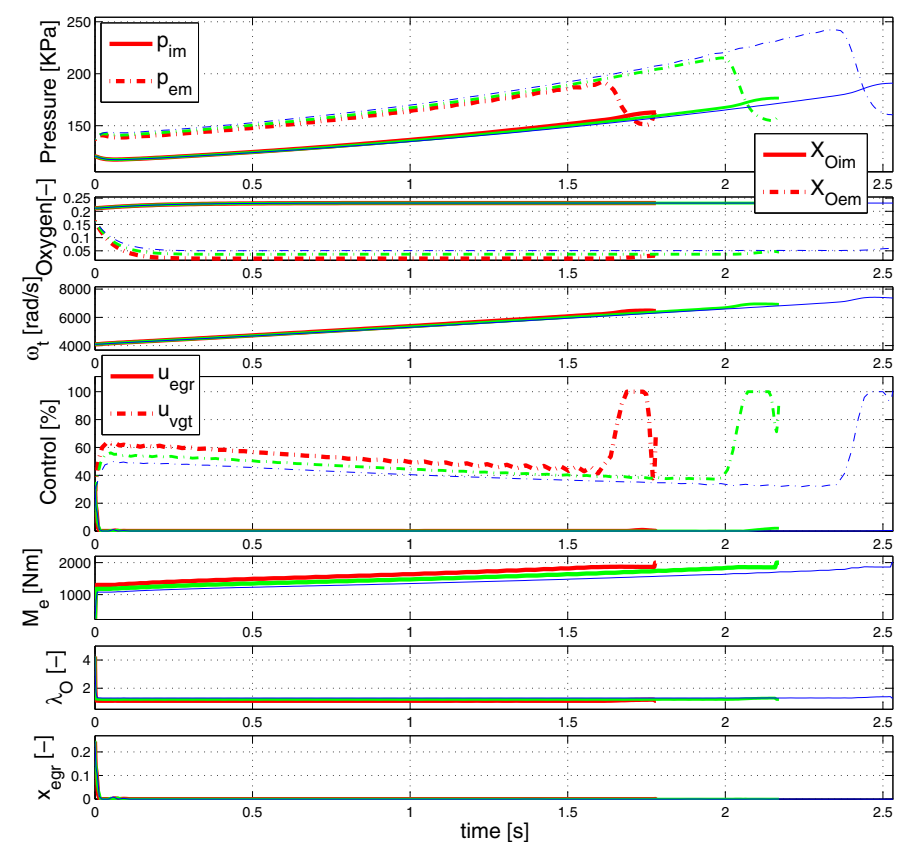

Fig. 6. Time optimal results without EGR limit. Red, green and blue are respectively $\lambda_{\mathrm{O}, \min }=$ $[1.1,1.2,1.3]$.

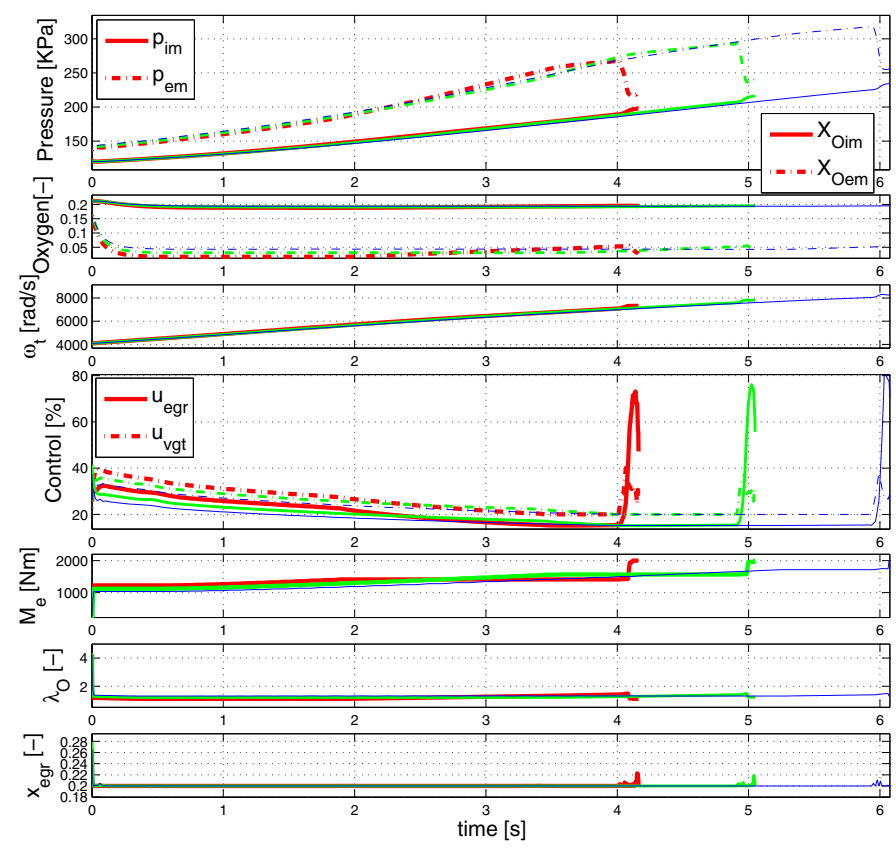

Fig. 7. Time optimal results for $x_{\text {egr,min }}=0.20$. Red, green and blue are respectively $\lambda_{\mathrm{O} \text {,min }}=[1.1,1.2,1.3]$.

or less EGR fraction is required for both fuel and time optimal results.

Figure 9 shows the transient time and the fuel consumption as function of $\lambda_{\mathrm{O} \text {,min }}$ and $x_{\text {egr,min }}$ for the fuel optimal case. The same is depicted in Figure 10, but for the time optimal case. Observing the figures one can see that fuel consumption and transient time increase almost exponentially with $\lambda_{\mathrm{O}, \min }$ and $x_{\mathrm{egr}, \min }$ for both time and fuel optimal controllers. This is also observed in figures 5 and 8 where the transient time increase for the highest 


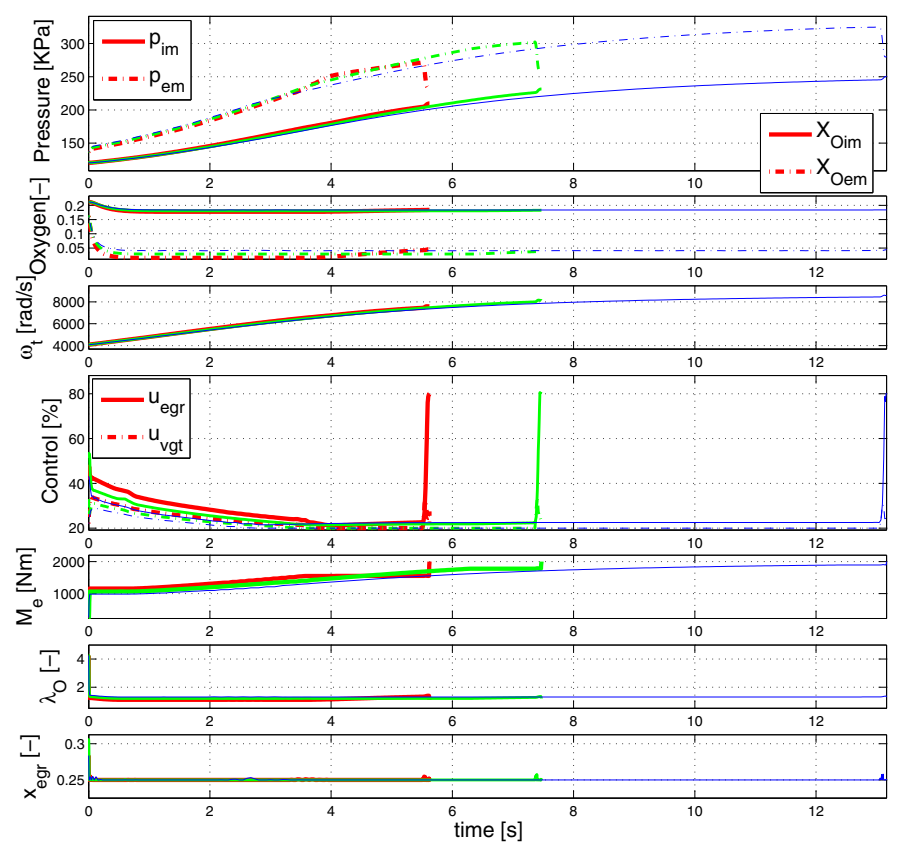

Fig. 8. Time optimal results for $x_{\text {egr,min }}=0.25$. Red, green and blue are respectively $\lambda_{\mathrm{O}, \min }=[1.1,1.2,1.3]$.
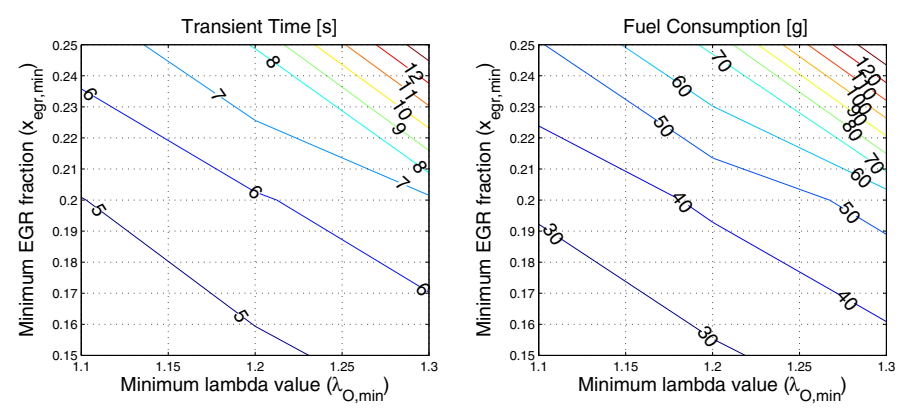

Fig. 9. Transient time and fuel consumption as function of $\lambda_{\mathrm{O} \text {,min }}$ and $x_{\mathrm{egr}, \min }$ for the fuel optimal case.

lambda is much higher than for the previous one. From Figures 9 and 10 one can observe that for $x_{\text {egr,min }}=0.25$ and $\lambda_{\mathrm{O}, \min }=1.3$, the fuel and time optimal results are very similar. This can also be seen by comparing Figures 5 and 8. The time optimal case requires $13.16 \mathrm{~s}$ and $143.03 \mathrm{~g}$ while the fuel optimal case requires a slightly higher time $13.72 \mathrm{~s}$ and a bit lower fuel consumption $141.44 \mathrm{~g}$. This happens because both solutions are at the limits of the smoke limiter and EGR fraction requirement during a long part of the transient, thus both achieve the final intake pressure with a very similar control strategy.

\section{CONCLUSION}

Optimal control of torque transients in a diesel engine with EGR and VGT systems is studied. The OCP is solved for a diesel engine model, which has the typical system nonminimum phase behaviors, overshoots and sign reversals. Due to the high complexity of the model, solving the problem can be tough. A strategy for how to proceed is presented, first the model complexity is decreased to produce initialization data. Then the problem constraints are progressively included to ease the solution convergence. A steady state study at the transient's end torque shows
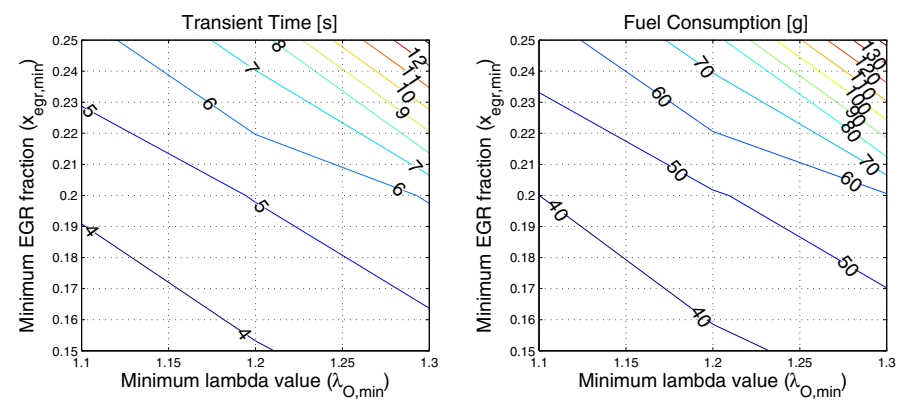

Fig. 10. Transient time and fuel consumption as function of $\lambda_{\mathrm{O} \text {,min }}$ and $x_{\mathrm{egr} \text {,min }}$ for the time optimal case

the importance of the intake manifold pressure for the maximum EGR fraction, these results are used to set feasible lower limits for $\lambda_{\mathrm{O}}$ and $x_{\mathrm{egr}}$

Time and fuel optimal results for the studied transient are presented explaining the controller strategy depending on different problem constraints. It is shown that $\lambda_{\mathrm{O}, \min }$ has a big effect on the transient duration but a minor effect on the control signals' shape. On the other hand, $x_{\text {egr,min }}$ has a big influence on the control signals. Finally a comparison between the time and the fuel optimal solutions shows that for high lambda and EGR values the control strategies become almost identical and both results achieve similar fuel consumption and transient time values. Future work is intended to include variable engine speed by studying segments of the WHTC.

\section{REFERENCES}

Ekdahl, A. (2005). Transient control of variable geometry turbine on heavy duty diesel engines. In Conference on Control Applications. IEEE.

Garg, D., Patterson, M., Hager, W.W., Rao, A.V., Benson, D.A., and Huntington, G.T. (2010). A unified framework for the numerical solution of optimal control problems using pseudospectral methods. Automatica, 46(11), $1843-1851$.

Kolmanovsky, I., Nieuwstadt, M., and Moraal, P. (1999). Optimal control of variable geometry turbocharged diesel engines with exhaust gas recirculation. In International Conference. IEEE/ASME.

Nieuwstadt, M., Moraal, P., Kolmanovsky, I., Stefanopoulou, A., Wood, P., and Widdle, M. (1998). Decentralized and multivarable designs for egr-vgt control of a diesel engine. In IFAC Workshop, Advances in Automotive Control. IFAC-AAC.

Sivertsson, M. and Eriksson, L. (2012). Time and fuel optimal power response of a diesel-electric powertrain. In IFAC Workshop on Engine and Powertrain Control, Simulation and Modeling. E-COSM'12.

TOMLAB (2010). PROPT - Matlab Optimal Control Software. URL http: //www. tomdyn.com/.

Wahlström, J. (2009). Control of EGR and VGT for Emission Control and Pumping Work Minimization in Diesel Engines. Ph.D. thesis, Linköping University.

Wahlström, J. and Eriksson, L. (2011). Modelling diesel engines with a VGT and EGR by optimization of model parameters for capturing non-linear system dynamics. Proceedings of the Institution of Mechanical Engineers, Part D, Journal of Automobile Engineering, 225(7), 960-986. 